\title{
Research of MCU ultrasonic range finder
}

\author{
Shuqing Ma ${ }^{1, a}$, Qingzhu Yan ${ }^{2, b}$, Chuanjun, Wang ${ }^{3, c}$ \\ 1 Jilin province changchun kaixuan road 3050jilin teachers college engineering college of electrical \\ engineering, China \\ 2 Changchun modern business vocational school Changchun jilin,China \\ 3Changchun modern business vocational school Changchun jilin,China \\ Aemail1154391951@qq.com
}

Key words: MSC - 51 (SCM); Ultrasound; The LED

Abstract: ultrasonic ranging system USES AT89C51 as the main controller, the dynamic scanning method is used to implement LED digital tube digital display, complete the ultrasonic driving signal with single-chip microcomputer timer. Ultrasonic directivity is strong, energy consumption slow, in the medium transmission distance is farther, thus the ultrasonic often used for distance measurement.

\section{The introduction}

Ultrasonic technology is a physical, electronic, mechanical, and material science on the basis of general technology, one of the bunching, directional, reflection, the projection features. Ultrasonic directivity is strong, energy consumption slow, in the medium transmission distance is farther, thus the ultrasonic often used for distance measurement.

In the industrial field, ultrasonic ranging is a mature new and high technology, ultrasonic ranging technology has many advantages:

(1) to detect quickly, convenient;

(2) simple calculation, easy to achieve real-time control;

(3) can be fixed point and continuous range, and can easily provide telemetry, or remote control signal;

(4) measurement with no direct contact with the object to be tested, to be able to clear and steadily display measurement results;

(5) compared with the laser ranging technology, ultrasonic ranging method is simple and low cost;

(6). Ultrasonic ranging technology can choose as gas, liquid or solid, sound transmission medium, thus has a strong environment adaptability.

\section{Ultrasonic ranging principle}

Transmitters of ultrasonic in nu of speed in air, in the object to be tested is reflected back, by the receiver, the round-trip time for $t$, by the $s=n / 2$ can calculate the distance of the object to be tested. Because ultrasound is also a kind of sound waves, the sound velocity $\mathrm{v}$ is associated with temperature, the following table lists the different temperature the speed of sound. When use, if the temperature change is not big, the velocity of sound can be thought of as basic same. If the ranging accuracy is very high, it should be corrected by the method of temperature compensation.

MCU signal that $40 \mathrm{KHZ}$, after amplification by ultrasonic transmitter output; Ultrasonic receiver will receive the ultrasonic signal by the amplifier amplification and detection using phase-locked 
loop circuit processing, start the SCM interrupt routine, measured time is $t$, again by the discriminant, calculation software, it is concluded that distance and LED display.

\section{The working process of the system}

\section{Ultrasonic receiving, amplifying circuit}

We know that the bandwidth of the op-amp 741 and anti-interference ability is strong, but in order to prevent to produce noise, so in the design of the ultrasonic signal amplification circuit, amplifying circuit design becomes a two level in phase ac amplifier, through parameter Settings, each level zoom in close to 20 times, so after two stage amplifier, the received ultrasonic signal is magnified is almost 400 times, completely subsequent detection circuit can be detected. Ultrasonic receiving circuit composed of mu A741 operational amplifiers, etc.

2. Signal filter circuit

This signal filter circuit can play a very important role in the whole circuit, through the appropriate adjustments, can effectively filter out due to the interference of the ultrasonic signal into the ultrasonic receiving system, thus greatly improving the anti-interference circuit.

3. Signal shaping circuit

When the received signal from the signal selection circuit after is a very irregular square wave signals, we hope to get a best pulse signal, therefore, also joined in the subsequent level circuit level by U1B, U1C and signal shaping circuit composed of resistors R5, through this part of the circuit processing after then sent into single chip microcomputer processing operation.

\section{AT89C51}

AT89S51 is a low power consumption, high performance CMOS 8-bit microcontroller, the device adopts high density of ATMEL company, nonvolatile storage technology, compatible with standard MCS - 51 and 80 s51 pin structure, instruction system AT89S51 is 40 pin, 4 k Bytes Flash memory on chip program, 128 Bytes of data random access memory (RAM), 32 external bidirectional input/output (I/O), five interrupts nested interrupt priority level 2 layer, two 16 bit programmable timer counter, two full duplex serial communication port, watchdog (WDT) circuit, on-chip oscillator clock.

\section{Algorithm design of ultrasonic range finder}

Emit ultrasonic ultrasonic transmitter to a certain direction, in the moment of timing starts at the same time, the ultrasonic wave in air, run into obstacles on the way to return immediately, ultrasonic receiver receive the reflected wave will immediately stop timing. The ultrasonic velocity in air is $340 \mathrm{~m} / \mathrm{s}$, according to the timer to record time $\mathrm{t}$, you can calculate the launch is apart from the obstacles of distance ( $\mathrm{s}$ ), namely: $\mathrm{s}=340 \mathrm{t} / 2$. This is the time difference ranging method. Ultrasonic ranging principle is to use the ultrasonic velocity in air is known, measuring the sound waves reflected time after launch to meet obstacles, according to the transmitting and receiving time to calculate the launch point to the actual distance of obstacles. Therefore, ultrasonic ranging principle and principle of radar is the same. Range of the formula is expressed as: $\mathrm{L}=\mathrm{C} * \mathrm{~T}$ type $\mathrm{L}$ for measuring distance in length; $\mathrm{C}$ for the ultrasonic velocity in the air; $\mathrm{T}$ for measuring distance transmission time ( $\mathrm{T}$ to receive half of the time value) for launch. 


\section{The analysis of the main program design}

Software is divided into two parts, the main program and interrupt service routine. The main program to complete the initialization, the control of various ultrasonic transmitting and receiving the order. Timer interrupt service subroutine to complete three direction ultrasonic launch by turns, external interrupt service subroutine of time value read out, the distance calculation, the result output, etc.

\section{The temperature sensor of debugging}

DS18B20 need only received a controller (MCU) of an I/O port, because of the single bus to open so I need external leakage a pull up resistance of $4.7 \mathrm{~K}$. If you want to use parasitic works, as long as VDD power supply pins with single bus in parallel. But in programming, parasitic works will have some special request for the state of the bus.

\section{Range of display circuit design}

LED digital display tube with two kinds, one kind is a total of anode digital tube, the interior is connected by eight anode of light-emitting diodes; Digital tube, the other is a common cathode inside it is composed of four anodes connected light emitting diode. The principle of which is different but the same function.

Because the single chip microcomputer Bunsen has strong ability of logic control, so the dynamic scanning software decoding is not complicated. And software decoding its decoding logic can be set in a random programming, without being limited by the hardware decode logic. By using dynamic scanning software decoding way can greatly simplify the hardware circuit structure, reduce the system cost. It USES time-sharing method take turns to control the display COM port, make each display took turns to light. In take turns to light up the scanning process, each display light up time is very short, but due to the phenomenon of persistence of vision and the afterglow effect of the light-emitting diodes (leds), gives the impression of is a set of stable display data. Static display data is stable and takes up less CPU time, but every display unit needs separate display driver circuit, using less hardware circuit; Dynamic display need CPU time to refresh the data display device, display data has the light flashes, the amount of CPU time is much, but using less hardware, circuit board space can be saved.

\section{Range of display circuit design}

Because the single chip microcomputer Bunsen has strong ability of logic control, so the dynamic scanning software decoding is not complicated. And software decoding its decoding logic can be set in a random programming, without being limited by the hardware decode logic. By using dynamic scanning software decoding way can greatly simplify the hardware circuit structure, reduce the system cost. It USES time-sharing method take turns to control the display COM port, make each display took turns to light. In take turns to light up the scanning process, each display light up time is very short, but due to the phenomenon of persistence of vision and the afterglow effect of the light-emitting diodes (leds), gives the impression of is a set of stable display data. Static display data is stable and takes up less CPU time, but every display unit needs separate display driver circuit, using less hardware circuit; Dynamic display need CPU time to refresh the data display device, display data has the light flashes, the amount of CPU time is much, but using less hardware, circuit board space can be saved. 


\section{The design of the ultrasonic transmission circuit}

Transmission circuit is mainly made up of 74 1s04 and ultrasonic transducer, a microcontroller P1.0 port $40 \mathrm{KHZ}$ square wave output signal path level after the reverser to ultrasonic transducer one electrode, after another through the two levels of reverser to ultrasonic transducer, another electrode with the push-pull forms the square wave signal to the ultrasonic transducer can improve the emission intensity at both ends.

\section{The design of the detection circuit}

Photoelectric sensor detection circuit can be used to make objects. But photoelectric sensor can't detect transparent objects. Infrared sensor in detecting objects need to have as humans and animals have certain temperature difference with the surrounding this condition. Ultrasonic sensors are not restricted by these conditions, the transparent or other objects can detect. Ultrasonic sensors to detect objects have directly reflect detection method and detection method.

\section{Improve the precision of the scheme and system design}

The analysis of the effect of temperature on ultrasonic velocity, the ultrasonic echo detection of ultrasonic transmission time and the effect of ultrasonic sensor by the influence of pulse voltage accuracy of the test. On this basis, the design of the ultrasonic digital rangefinder. The experimental results show that pay attention to the above three factors can improve the precision of ultrasonic distance measurement.

Ultrasonic ranging because of its in use is not affected by factors such as light, electromagnetic field, the color, and simple structure, lower cost, in the robot obstacle avoidance and positioning, reverse, reservoir level measurement and so on have been widely used. In principle, ultrasonic distance measurement with pulse echo method, resonance method and frequency difference method. The commonly used method of pulse echo ranging, its principle is emit ultrasonic ultrasonic sensor, analyte spread in the air, after reflection by the ultrasonic sensor to receive reflected pulse, measure the ultrasonic pulse from transmitting to receiving time ts, on the premise of known supersonic velocity $\mathrm{Cs}$, can be calculated by measuring the distance of the $\mathrm{S}$, namely: $\mathrm{S}=\mathrm{CT} / 2$

\section{conclusion}

The principle of ultrasonic distance measurement is the use of ultrasonic transmission and reception, according to the ultrasonic transmission time to calculate the propagation distance. Practical ranging method has two kinds, one kind is at opposite ends of the measured distance, one end of the launch, the other end receives the direct wave, suitable for height measure; One is transmitted wave is reflected by the object after receiving the reflected wave method, is suitable for the range finder. This design adopts the reflection wave method.

\section{References}

[1] Yigang zhang Peng Xiyuan Tan Xiaoyun compiled, MCS - 51 single chip microcomputer application design, Harbin industrial university press, 1997.

[2] Wang Wujiang. Commonly used integrated circuit quick manual. Beijing: metallurgical industry press, 2004.12.

[3] Li Guangdi Zhu Yuexiu Xiu-shan wang By single chip microcomputer based, Beijing university of aeronautics and astronautics press, July 2001, revised edition 
[4] xin-min pan Wang Yanfang compiled, micro computer control technology, the electronic industrial press, in January 2003, the first edition

[5] chenrui Wang Xiaoliang Wu hao Chao-ping zhang compiled, the design of the sensor system and the maintenance, shandong science and technology press, February 2003, Cao Chengzhi Liu Baoji Wei Yongguang, single-chip microcomputer control frequency conversion system, power electronic technology, in 1999, the second phase

[6] Wu Zhongzhi Wu Jialin compiled, MCU application manuals, mechanical industry publishing house, in April 2003, the second edition

[7] king of zhi Wang ms: modern sensor technology [M]. Electronic industry press. 1995.331-335

[8] Sun Hanfang Xu Aiqing: MCS a 51/96 series single chip microcomputer principle and application (revised edition) [M]. Beijing: Beijing university of aeronautics and astronautics press. 2002.46-170-2

Jin is Wang Jianqin [9] road Shao-guo Yang Zhao Ke Zhao too fly: ultrasonic rangefinder design [J]. Journal of sensor technology. 2002

[10] xiu-qin CAI. 8254 in the application of improving precision of ultrasonic ranging system [J]. Journal of electronic science and technology, 2005 